\title{
Tomographic Reflection to Merge Ultrasound Images with Direct Vision
}

\author{
George D. Stetten, M.D., Ph.D \\ U. Pittsburgh - CMU Robotics Inst. \\ george@stetten.com
}

\author{
Vikram S. Chib \\ U. Pittsburgh - CMU Robotics Inst. \\ vchib@andrew.cmu.edu
}

\author{
Robert J. Tamburo \\ University of Pittsburgh \\ rjtst21@pitt.edu
}

\begin{abstract}
Tomographic reflection is a method that may be used to merge the visual outer surface of a patient with a simultaneous ultrasound scan of the patient's interior. The technique combines a flat-panel monitor with a halfsilvered mirror such that the image on the monitor is reflected precisely at the proper location within the patient. In this way, the ultrasound image is superimposed in real time on the view of the patient along with the operator's hands and any invasive tools in the field of view. Instead of looking away at an ultrasound monitor, the operator can manipulate needles and scalpels with direct hand-eye coordination. Invasive tools are visible up to where they enter the skin, permitting natural visual extrapolation to targets in the ultrasound slice. Tomographic reflection is independent of viewer location, requires no special apparatus to be worn by the operator, nor any registration of the patient.
\end{abstract}

\section{Introduction}

Percutaneous ultrasound-guided intervention encompasses a wide range of procedures in clinical medicine [1-3] , including biopsy of liver [4] , breast [5] , lymph nodes [6-8], and thyroid [9], as well as central venous access [10] and various musculoskeletal interventions [11]. In such procedures, a needle is typically constrained by a guide attached to the transducer so that the entire length of the needle remains visible within the plane of the ultrasound scan. The operator must look away from the patient at the ultrasound display and employ a displaced version of hand-eye coordination. These constraints have motivated research into developing techniques to visually merge ultrasound with real-world views in a more natural and unconstrained manner.

Fuchs, et al., have experimented with a head mounted display (HMD) following two distinct approaches in what they call augmented reality. In the first approach, they optically combined a direct view of the patient with ultrasound images using small half-silvered mirrors mounted in the HMD [12]. More recently, they have replaced direct vision with miniature video cameras in the HMD, displaying merged video and ultrasound images on miniature monitors in the HMD. This second approach permits greater control of the display, although it introduces significant reduction in visual resolution [1315]. In both cases, the HMD and the ultrasound transducer must be tracked for an appropriate perspective to be computed for the ultrasound images. Headmounted displays, in general, restrict the operator's peripheral vision and freedom of motion.

In related work, DiGioia, et al., have merged realworld images with computerized tomography (CT) data, while achieving a reduction in the apparatus that the operator must wear $[16,17]$. In their system, called image overlay, a large half-silvered mirror is mounted just above the patient with a display monitor fixed above the mirror. Images of CT data on the monitor are reflected by the mirror and superimposed on the view of the patient through the mirror. The operator need only wear a small head-tracking optical transmitter so that a correct perspective of the three-dimensional CT data can be rendered. Special glasses are needed only if stereoscopic visualization is desired. A tracking device must be attached to the patient to achieve proper registration between the rendered $\mathrm{CT}$ data and the patient.

We have modified DiGioia's approach and applied it to ultrasound, with significant simplification. By restricting ourselves to a single tomographic slice in real time (i.e. ultrasound), and strategically positioning the transducer, the mirror, and the display, we have eliminated the need for tracking either the observer or the patient. This is possible because we are actually merging the virtual image in $3 \mathrm{D}$ with the interior of the patient. Ultrasound produces a tomographic slice within the patient representing a set of $3 \mathrm{D}$ locations that lie in a plane. The image of that tomographic slice, displayed at its actual size on a flat panel display, may be reflected to occupy the same physical space as the actual slice within the patient. If a half-silvered mirror is used, the patient may be viewed through the mirror with the reflected 
image of the slice superimposed, independent of viewer location. The reflected image is truly occupying its correct location within the patient and does not require a particular perspective to be rendered. Therefore we have adopted the term tomographic reflection rather than image overlay.

\section{Materials and Method}

To accomplish tomographic reflection, certain geometric relationships must exist between the slice being scanned, the monitor displaying the slice, and the mirror. As shown in Fig. 1, the mirror must bisect the angle between the slice and the monitor. On the monitor, the image must be correctly translated and rotated so that each point in the image is paired with a corresponding point in the slice to define a line segment perpendicular to, and bisected by, the mirror. By fundamental laws of optics, the ultrasound image will thus appear at its physical location, independent of viewer position.

The above concept was implemented using an apparatus consisting of an ultrasound transducer (3.5 MHz. curvilinear), a flat panel video monitor (Samsung, $150 \mathrm{MP}$ ), and a half-silvered mirror (32" x 18"). The relative positioning of these components is shown in Fig. 2A. The ultrasound transducer and the flat panel monitor were mounted on opposite sides of the mirror, with each fixed at an angle of $60^{\circ}$ relative to the mirror. The video image produced by the ultrasound scanner (Acoustic Imaging 5200B) was fed through a graphics computer $\left(\mathrm{SGI}_{2}\right)$ and displayed on the flat panel monitor. The purpose of the graphics computer was to scale, rotate and translate the image so that its reflection merged properly with the physical slice. The computer accomplished this video processing continually at a frame rate of approximately 5 frames/sec and a latency of approximately 0.2 seconds.

Calibration of the apparatus was accomplished by visual inspection. First, with the transducer pointing into open air, and with the gain increased to display an "empty slice", the transducer was positioned so as to make the reflected image of the slice appear to emanate accurately from the head of the transducer (see Fig 2B). A strong sense of the location and orientation of the reflected image relative to the transducer permitted this to be accomplished with confidence. Various targets were then scanned and adjustments made to the scale, orientation, and translation on the graphics computer to accomplish visual alignment between the target and its reflected ultrasound image. The calibration, once established, remained effective for subsequent targets.

The apparatus was tested on a number of in vitro and in vivo targets, with the goal of establishing a proof of concept for the overall technique. Several examples are described in the following section. Photographs were taken from the point of view of the operator, i.e., looking through the mirror at the target with the ultrasound image

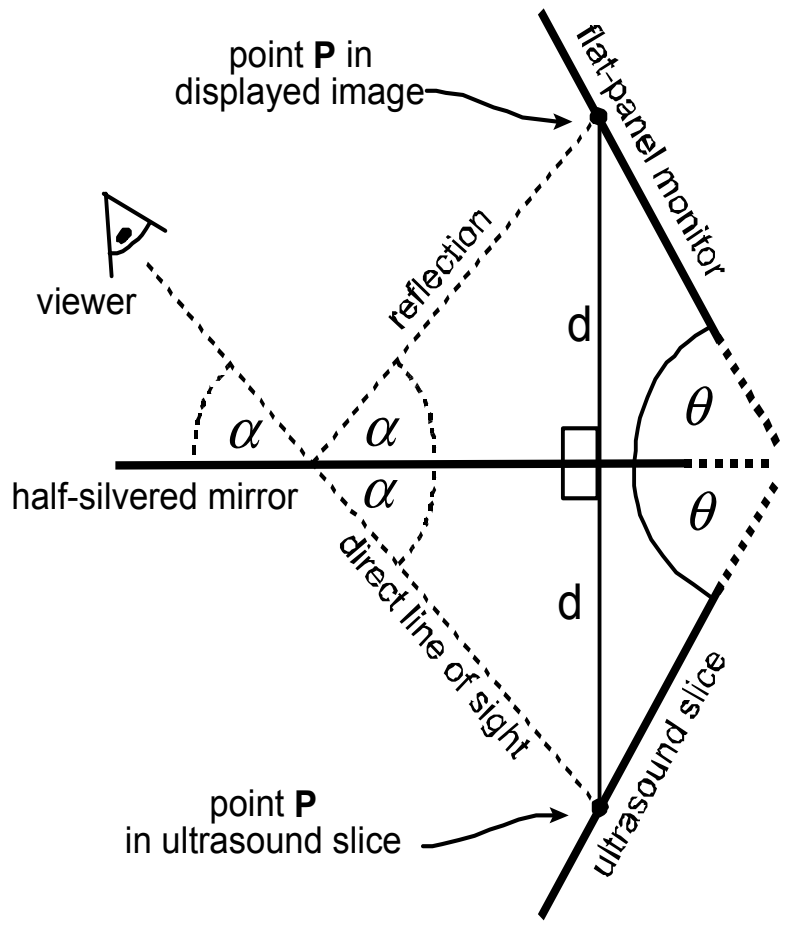

Figure 1. The half-silvered mirror bisects the angle $2 \theta$ between the ultrasound slice (within the target) and the flat-panel monitor. Point $\mathbf{P}$ in the ultrasound slice and its corresponding location on the monitor are equidistant from the mirror along a line perpendicular to the mirror (distance $=d)$. Because the angle of incidence equals the angle of reflectance (angle $=\alpha$ ) the viewer (shown as an eye) sees each point in the reflection precisely at its corresponding physical 3D location.

reflected in the mirror.

\section{Results}

The following examples demonstrate the efficacy of the technique and illustrate potential clinical applications. 


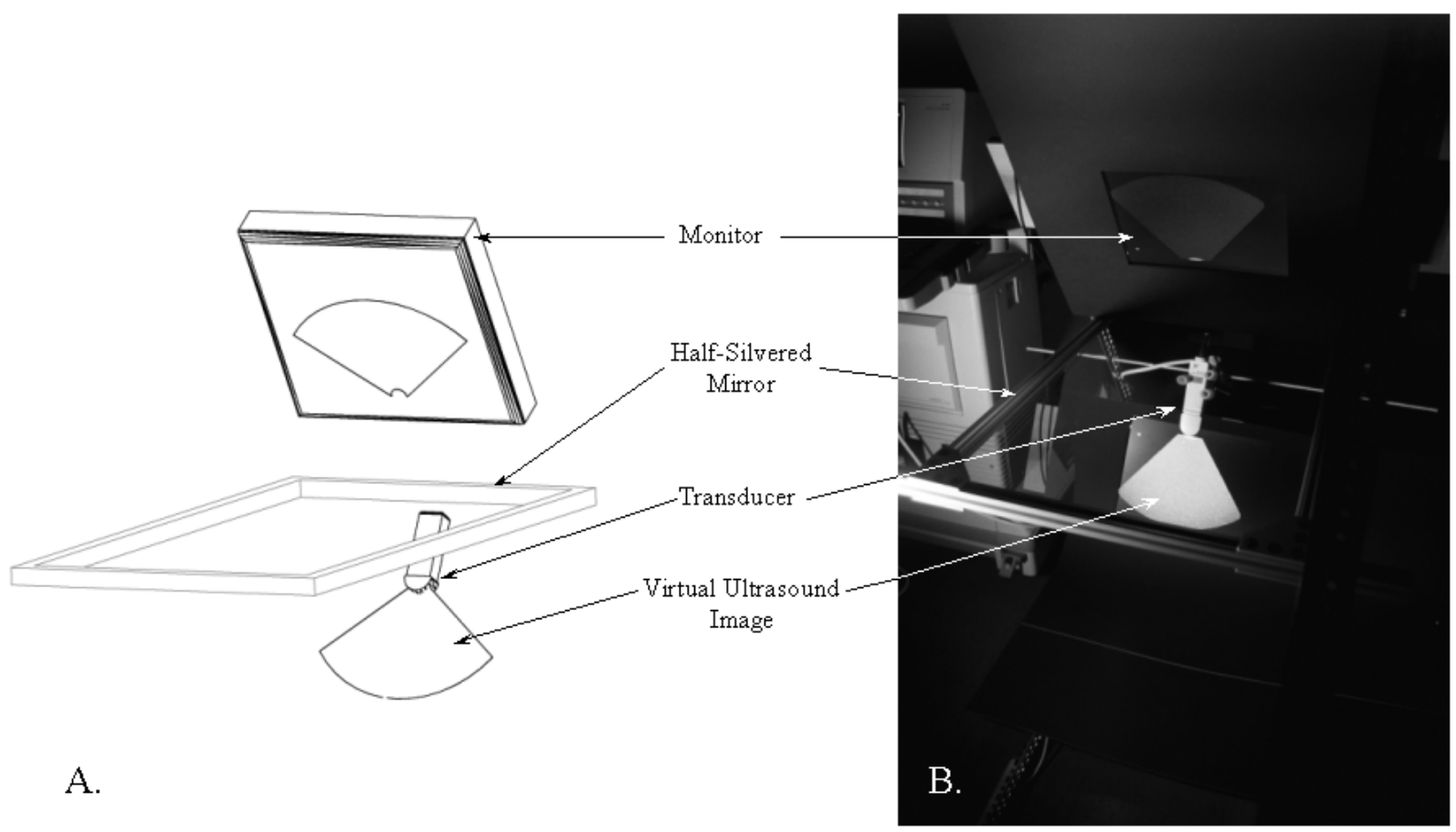

Figure 2. A. Schematic representation of the apparatus. A flat-panel monitor and an ultrasound transducer are placed on opposite sides of a half-silvered mirror such that the mirror bisects the angle between them. B. Photograph of apparatus with transducer in the open air generating an "empty slice".

In Fig 3, a human hand is seen with the transducer pressed against the soft tissue between the thumb and index finger. While not a common target for clinical ultrasound, the hand was chosen because it clearly demonstrates successful alignment. The external surfaces of the hand are located consistent with structures within the ultrasound image. The photograph cannot convey the strong sense, derived from stereoscopic vision, that the reflected image is located within the hand. This sense is intensified with head motion because the image remains properly aligned from different viewpoints. To one experiencing the technique first-hand, ultrasound targets within the hand are clearly accessible to direct percutaneous injection, biopsy or incision

In Fig. 4, a water-filled balloon is scanned. A protrusion into the balloon, which is produced by the operator's finger, is visible in the ultrasound image. Inside the balloon, a short piece of rubber tubing has been placed to simulate an artery or vein. The tube is seen in cross-section with the end of the operator's finger pressing against it. This demonstrates a combination of tactile and visual feedback that the operator could use to introduce an intra-vascular catheter. Unlike conventional ultrasound-guided intervention, in which the needle is restricted to lie within the slice, our system permits the tube to be targeted in cross-section thereby presenting its lumen as a convenient "bull's-eye" for catheter insertion.

\section{Discussion}

We have demonstrated a new method for combining human vision with ultrasound images in a natural and simple manner. Tomographic reflection is achieved without requiring any special apparatus to be worn by the operator or any tracking of the patient. Further, it is independent of viewer location and permits stereoscopic vision, in effect, to penetrate the skin with ultrasound.

The work to date is presented simply as a proof of concept. Rigorous calibration will be required to quantify and minimize error before clinical applications can be tested. In the present apparatus the ultrasound transducer is immobilized. This clearly presents difficulties for the operator accustomed to freely moving the transducer during an examination. We plan to address this problem by allowing the transducer to be hand-held while tracking its location. A combination of 
IEEE Proceedings of the Applied Imagery Pattern Recognition (AIPR) annual workshop, 2000, pp. 200-205.

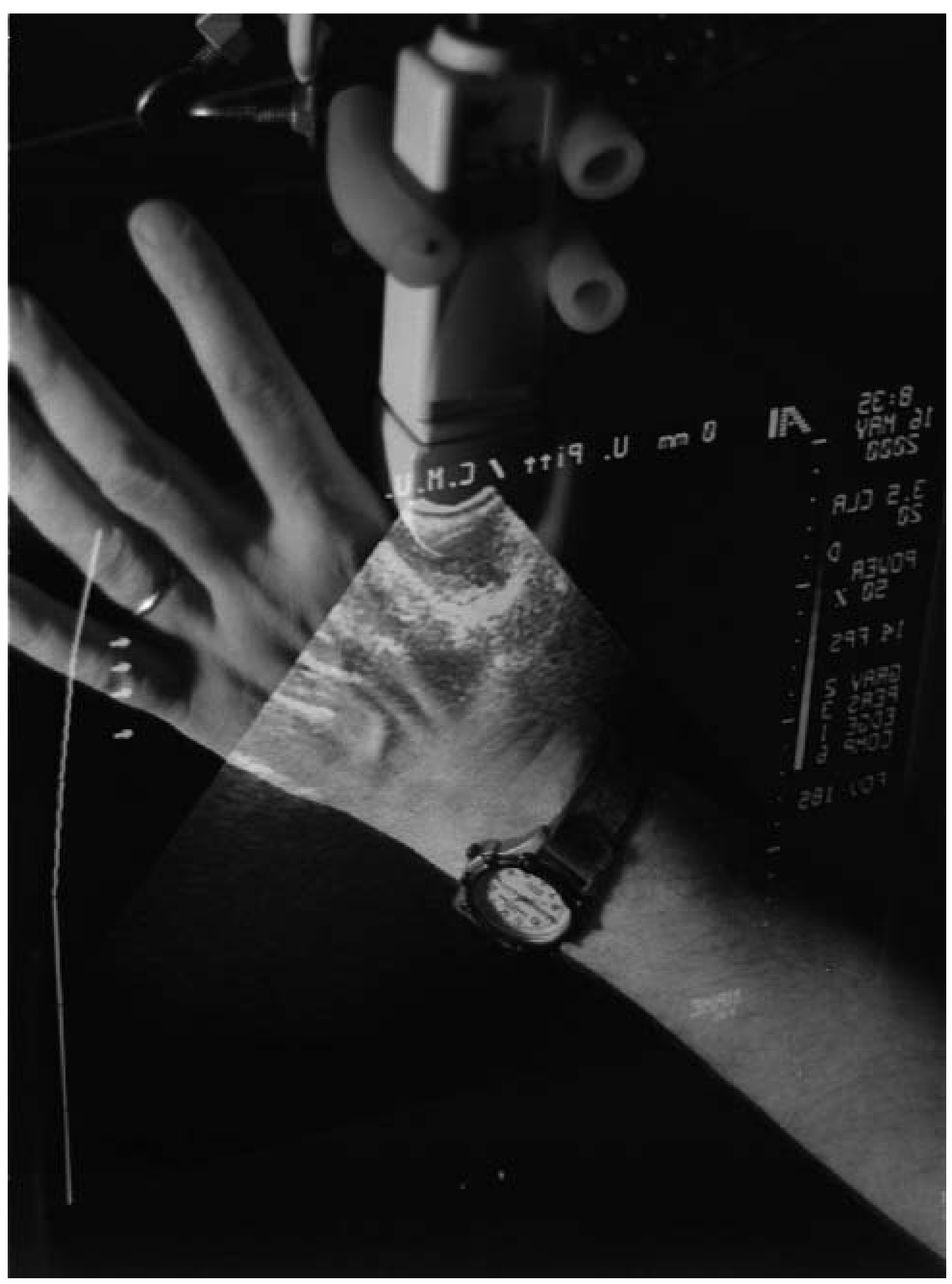

Figure 3. Photograph, from the viewpoint of the operator, showing a scan of a hand using the apparatus in Fig. 2. The reflected ultrasound image is merged with the direct visual image. 
IEEE Proceedings of the Applied Imagery Pattern Recognition (AIPR) annual workshop, 2000, pp. 200-205.

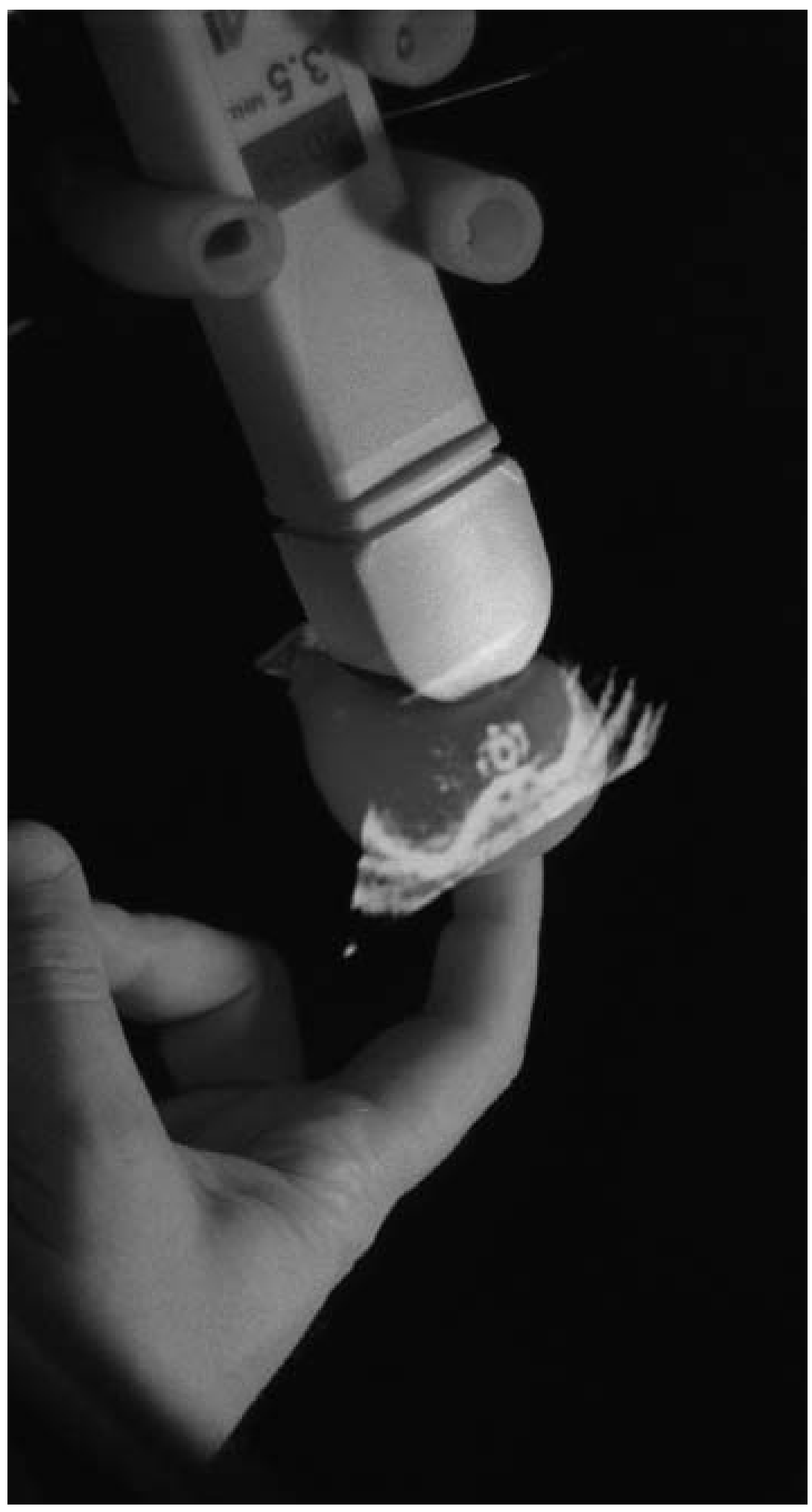

Figure 4. Scan of a fluid-filled balloon containing a short piece of rubber tubing (tubing is seen in cross-section) Operator's finger is seen pressing into the balloon against the tubing. 
IEEE Proceedings of the Applied Imagery Pattern Recognition (AIPR) annual workshop, 2000, pp. 200-205.

robotic manipulation of the monitor and graphical manipulation of the image would keep the reflected image properly aligned.

Superimposing ultrasound images on human vision may improve an operator's ability to find targets while avoiding damage to neighboring structures, while generally facilitating interpretation of ultrasound images by relating them spatially to external anatomy. As such, it holds promise for increasing accuracy, ease, and safety during percutaneous biopsy of suspected tumors, amniocentesis, fetal surgery, brain surgery, insertion of catheters, and many other interventional procedures.

\section{Acknowledgements}

This research was supported through a seed-fund grant from Carnegie Mellon University and a grant from the Whitaker Foundation to the Department of Bioengineering at the University of Pittsburgh. Valuable assistance and advice was received from Costa Nikou, Richard LaBarca, Robert Kucler, Reed McManigle, Mark Peterson, Joseph Maroon, and the Medical Robotics and Computer Assisted Surgery (MRCAS) Laboratory in the CMU Robotics Institute.

\section{References}

[1] G. Dodd, C. Esola, D. Memel, et al., Sonography: the undiscovered jewel of interventional radiology. Radiographics, 1996. 16: 1271-1288.

[2] D. Sheafor, E. Paulson, C. Simmons, et al., Abdominal percutaneous interventional procedures: comparison of CT and US guidance. Radiology, 1998. 207(3): 705-710.

[3] H. Holm and B. Skjoldbye, Interventional ultrasound. Ultrasound Med Biol, 1996. 22(7): 773-789.

[4] S. Shah, J. Mayberry, A. Wicks, et al., Liver biopsy under ultrasound control: implications for training. Gut, 2000. 46(4): 582.

[5] J. Liu, B. Fornage, B. Edeiken-Monro, et al., The Reliability of Ultrasound-Guided Core Needle Biopsy (US$C N B)$ in the Evaluation of Non-Palpable Solid Breast Lesions. Laboratory Investigation, 1999. 79(1): 26A.
[6] S. Gupta, C. Rajak, B. Sood, et al., Sonographically guided fine needle biopsy of abdominal lymph nodes: experience in 102 patients. J Ultrasound Med, 1999. 18(2): 135-139.

[7] D. Memel, G. Dodd, and C. Esola, Efficacy of sonography as a guidance technique for biopsy of abdominal, pelvic, and retropertoneal lymp nodes. AJR Am J Roentgenol, 1996. 167(4): 957-962.

[8] A. Fisher, E. Paulson, D. Sheafor, et al., Small lymph nodes of the abdomen, pelvis, and retroperitoneum: usefulness of sonographically guided biopsy. Radiology, 1997. 205(1): 185-190.

[9] G. Boland, M. Lee, P. Mueller, et al., Efficacy of sonographically guided biopsy of thyroid masses and cervical lymph nodes. AJR Am J Roentgenol, 1993. 161(5): 1053-1056.

[10] W. Fry, G. Clagett, and P. O'Rourke, Ultrasound-Guided Central Venous Access. Archives of Surgery, 1999. 134(7): 738-741.

[11] E. Cardinal, R. Chhem, and C. Beauregard, Ultrasoundguided interventional procedures in the musculoskeletal system. Raiol Clin North Am, 1998. 36(3): 597-604.

[12] R. Azuma, A Survey of Augmented Reality. A Survey of Augmented Reality, in Presence: Teleoperators and Virtual Environments, 1997. 6(4): 355-385.

[13] A. State, M. Livingston, W. Garret, et al. Technologies for Augmented Reality Systems: Realizing Ultrasound-Guided Needle Biopsies. in ACM SIGGRAPH. 1996. New Orleans, LA,

[14] H. Fuchs, A. State, E. Pisano, et al. Towards Performing Ultrasound-Guided Needle Biopsies from within a HeadMounted Display. in Visualization in Biomedical Computing. 1996. Hamburg, Germany.

[15] H. Fuchs, M. Livingston, R. Raskar, et al. Augmented Reality Visualization for Laproscopic Surgery. in MICCAI. 1998. Massachusetts Institute of Technology, Cambridge, MA, USA.

[16] A. DiGioia, B. Colgan, and N. Koerbel, Computer-Aided Surgery, in Cybersurgery, R. Satava, Editor. 1998, Wiley: New York. p. 121-139.

[17] M. Blackwell, F. Morgan, and A. DiGioia, Augmented Reality and Its Future in Orthopaedics. Clinical Orthopaedic and Related Research, 1998. 345: 111-122. 